\title{
Immediate Implant Placement in Maxillary Molars using Septa Dilatation with Threaded Expanders: 3 Years Follow-up and use of the Technique in Complex Cases
}

\author{
Cesar G Luchetti, Gregori M Kurtzman, Alicia E Kitrilakis, Daniel I Ostrowicz
}

\begin{abstract}
Challenges often confront the surgeon when placing implants into the maxillary arch due to lower bone density. This can be further complicated due to the proximetry of the maxillary sinus especially when immediate implant placement is contemplated at time of extraction. This article will review the use of rotary osteotomes for improvement of bone site density and quality to allow implant placement in the maxillary posterior.
\end{abstract}

Keywords: Implant, Immediate placement, Extraction site, Expansion, Grafting.

How to cite this article: Luchetti CG, Kurtzman GM, Kitrilakis $A E$, Ostrowicz DI. Immediate Implant Placement in Maxillary Molars using Septa Dilatation with Threaded Expanders: 3 Years follow-up and use of the Technique in Complex Cases. Int J Oral Implantol Clin Res 2012;3(3):138-145.

\section{Source of support: Nil}

Conflict of interest: None declared

\section{INTRODUCTION}

Immediate placement of dental implants has reported to reduce treatment times and provide better preservation of the alveolar ridge. ${ }^{1-3}$ This concept has limitations during replacement of teeth with multiple roots as found in molar positions. ${ }^{4}$ Additionally, the maxillary molar region also presents a lower bone density and the proximetry of the maxillary sinus. Many attempts have been made to place implants into extraction sockets in the maxillary posterior, while some advocate that the best treatment option is a delayed approach following extraction. Immediate options include placement of the implant in the palatal socket offaxis or modification of the palatal socket. ${ }^{5-7}$ In a previous published article in 2006, the author (Luchetti) presented a technique to place immediate implants in maxillary molar sites using the interseptal bone, which has shown better clinical results compared to the delayed approach after osseous healing. ${ }^{8}$

The original technique included the use of sharpened osteotomes and a partial thickness-rotated palatal flap to achieve primary closure. As evolution of the technique developed, during the past 4 years the author started to dilate the socket using threaded expanders. This proved to be a more comfortable procedure for the patient. The technique includes the use of a healing abutment to be placed at time of implant placement making the surgical procedure a one- stage approach. The first results at 1 year follow-up were presented in another article in $2009 .{ }^{9}$ In this new article, we are presenting the results at 3 years with the threaded expanders approach, and also showing that the technique can be used in challenging cases, as is the case that illustrates the article. The aim of this study was to improve a proven technique and to compare it to the original procedure in order to place immediate implants in maxillary molar region.

\section{MATERIALS AND METHODS}

\section{Case Selection}

The technique is specifically indicated in cases of tooth extraction necessitated by caries with minimal bone loss that does not extend into the furcal portion of the root. The more bone that has been maintained along with the septum of bone between the roots the easier the technique is to perform. Additionally, the anatomy of the roots plays an important role, with roots with more spread and thicker septa providing a more favorable situation.

If after expansion of the socket has been completed, should the expander have insufficient initial stability, which could serve as a reference of the possible stability of the implant, the procedure can be aborted; the socket graft and closed to be re-entered and a delayed placement can be performed 2 to 4 months later.

\section{Instruments}

The threaded expanders have a conical shape. The set is composed of five expanders with a sharpened terminal end plus an additional tool with a flat end which is used for sinus lifts (Microdent System, Spain). First two expanders (yellow and red) are for initiation of the site increasing the site from $1 \mathrm{~mm}$ diameter to 2.3 (yellow) or $3 \mathrm{~mm}$ (red). Expander number 3 (blue) is used for 3.3 to 3.75 implants. Expander number 4 (green) is used for 3.75 to 4.2 implants and expander number 5 (black) is used for $5 \mathrm{~mm}$ implants (Fig. 1).

The sequence is chosen according to the shape of the implant fixture being used and the density of the bone encountered. In most situations, it is recommended that tapered implants are utilized, and site development is stopped at the green expander (\#4). The implant shown in the case presented has a $3.4 \mathrm{~mm}$ diameter in the apical part 


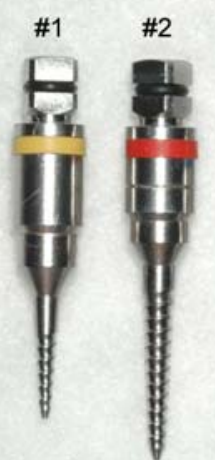

Coronal $2.3 \quad 3.0$

Apical 1.0

Fig. 1: The expanders indicating the diameter of the apical tip and the coronal aspect to be used for site development

and $5 \mathrm{~mm}$ diameter at the platform (NG implant - B\&W, Argentina). When soft bone (D4) is encountered as the bone has low density, the expansion is stopped after the green expander has been used and further expansion is accomplished when the implant is placed. If denser bone is encountered, the black expander (\#5) should be used to make the implant placement easier and decrease incidence of fracture of the buccal plate.

Threaded expanders are usually manually driven, using a thumb wrench (finger wheel) provided by the system or a Ratchet wrench. More recently, after our last article on the subject, we have incorporated the mechanically driven approach using a special adapter for the handpiece, which allows better control (Fig. 2). Anyway, when the advance of the expander meets greater resistance, a wrench must be used to generate greater torque and advance the expander to its desired depth.

One of the potential problems when using the expanders manually is the risk to tilt the preparation mesially due to the direction of the rotational forces applied. The use of the mechanical approach has simplified this.

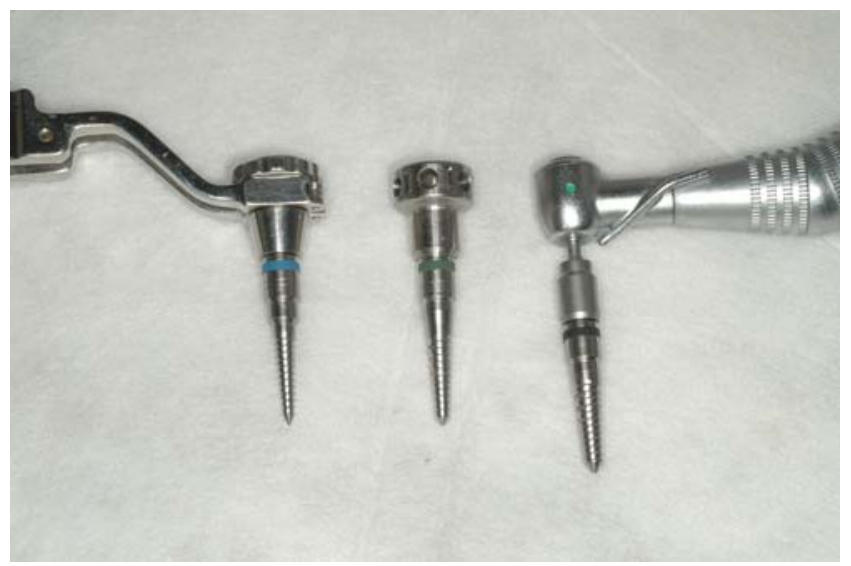

Fig. 2: Different options to drive the expanders are shown in the photo. From left to right: Ratchet wrench, finger wheel and handpiece

\section{CLINICAL TECHNIQUE}

The initial step of the technique is an atraumatic extraction. This is common in all the cases when an immediate placement is planned, but is very important that the septa are preserved during the extraction process. If during extraction the septa are lost, then the technique cannot be performed. If necessary, the tooth can be sectioned and the individual roots extracted one by one. Following the extraction, a carefully examination and debridement of the socket is performed, using curettes and the socket is decontaminated with a $2 \%$ citric acid gauze sponge packed into the socket for 1 minute.

Implants are immediately placed using a technique of septal dilatation using threaded expanders. The procedure begins with the use of a $1 \mathrm{~mm}$ diameter pilot drill to create the initial preparation for the expanders to follow. The procedure continues through the use of threaded expanders in a sequence of increasing diameters to a size smaller than the intended implant diameter to be placed. Following this, an implant $5 \mathrm{~mm}$ wide with a length of $10 \mathrm{~mm}$ and a tapered fixture shape is placed. A healing abutment is placed on the implant. The residual spaces of the socket surrounding the fixture are grafted and the soft tissues are sutured to achieve tight closure around the healing abutment.

The differences with the previous technique can be divided in two areas: Instrumentation and management of the soft tissue. The previous technique used osteotomes, which did not require the use of a starter drill, had good control of the direction of the osteotomy but is more aggressive for the patient. Regarding soft tissues, the original technique was developed as two stages, which included a partial thickness-rotated palatal flap to close the site. This was developed based on concepts at that time, which held to the concept of a two-stage surgical approach. The main problem with this approach was that the donor area for the palatal flap had an increased morbidity and presented greater discomfort for the patient. The current technique modified the procedure to a single stage, using a healing abutment at implant placement with approximation of the soft tissues surrounding the healing abutment. This has two major advantages. First, no palatal rotated flap is needed to close the site, and second, no additional surgery is necessary to uncover the implant. However, in complex cases we may still want to close completely the soft tissue and use a two-stage approach. In such cases, we use now two small vertical incisions in the buccal side; release the periosteum and move the flap coronally. This has proved to be effective and is more comfortable for the patient compared to the rotated palatal flap. 


\section{CASE REPORT}

A patient, 55-year-old female was presented with an upper molar with extended decay and a big periapical lesion invading the maxillary sinus (Figs 3 and 4).

The molar was not restorable, so extraction was recommended with immediate placement of an implant followed by a healing period prior to restoration of the implant.

The molar was atraumatically extracted to preserve the bone at the buccal crest as well as the furcation and the periapical lesion was carefully eliminated (Figs 5 and 6).

After first review of the socket, the suspected communication with the maxillary sinus was confirmed and also a dehiscence in the buccal wall was noticed.

A small flap was raised in order to get better access for the cleaning procedure, which was performed using manual curettes and citric acid 2\% imbibed in gauze for 1 minute (Fig. 7).

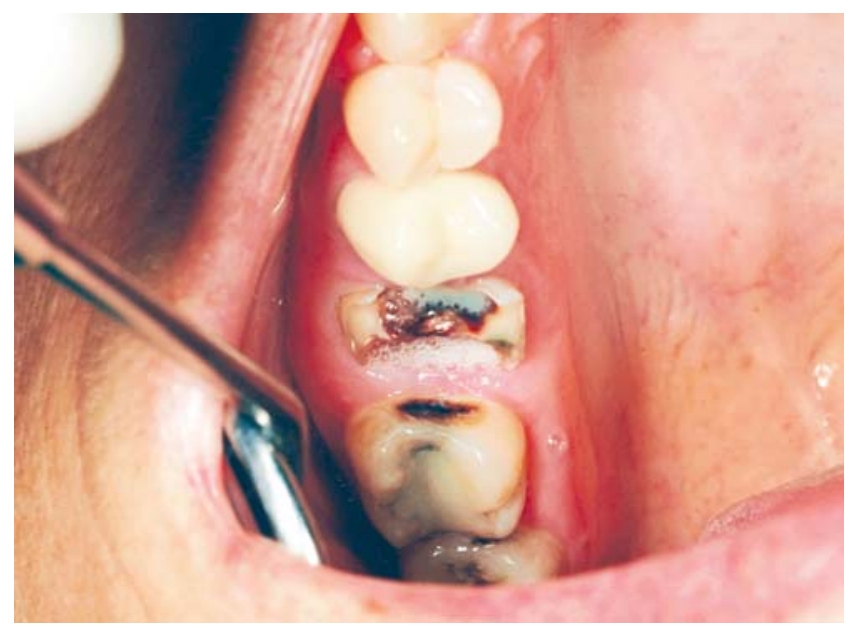

Fig. 3: Maxillary first molar demonstrating coronal breakdown

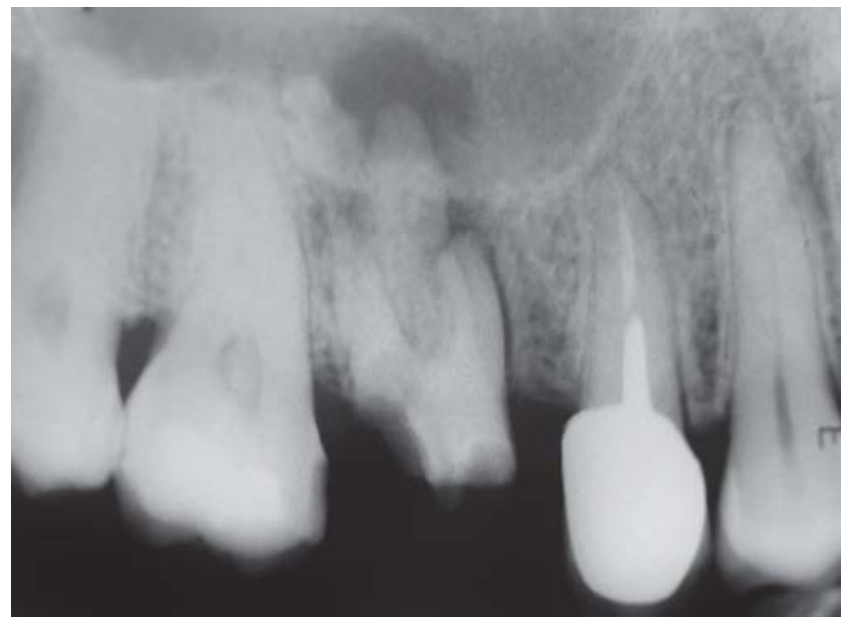

Fig. 4: Maxillary first molar demonstrating coronal breakdown and a big periapical lesion invading the maxillary sinus
Implant site preparation was done using the threaded expanders technique in order to achieve the septa dilatation. A pilot drill was used in a surgical implant handpiece to

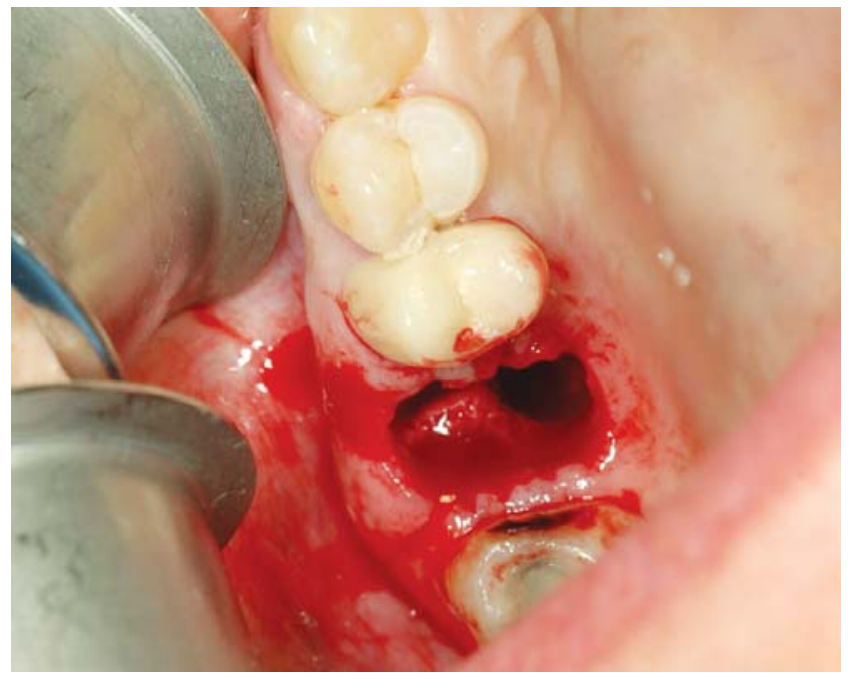

Fig. 5: Conservation of the socket after extraction

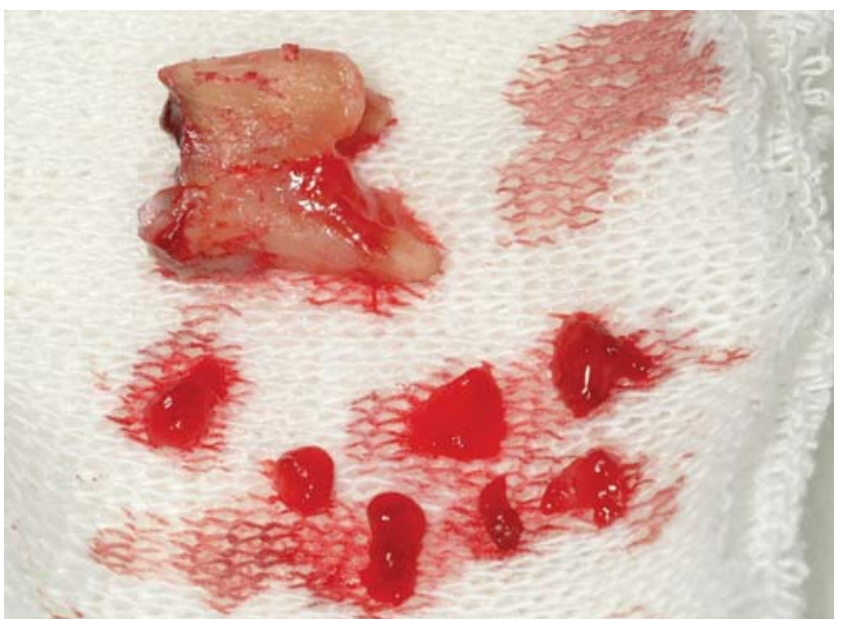

Fig. 6: Molar extracted together with the periapical lesion

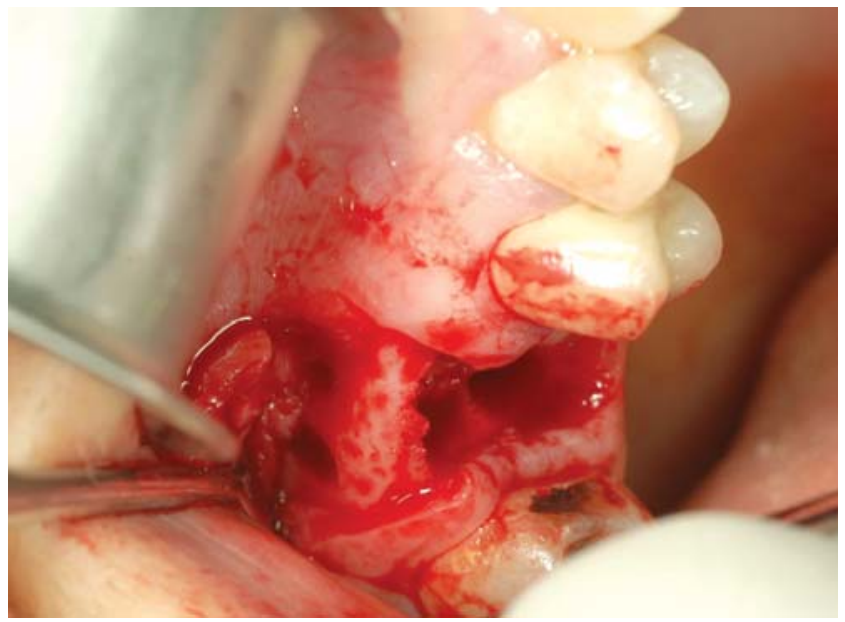

Fig. 7: Small flap was raised, showing the dehiscence in the buccal wall 
initiate the osteotomy at the center of the septal bone of the furcation. Subsequently, the expanders are used to laterally expand the sockets bone and complete the osteotomy in preparation for implant placement (Fig. 8). Final expansion of the site is performed with the implant (NG Implant, B\&W, Argentina) which is slowly placed into the furcation osteotomy (Fig. 9).

A collagen membrane (Collagene AT, Italy) was placed in the apical part of the remaining palatal socket to help the maxillary sinus communication closure (Figs 10 and 11). Then, a bone substitute (Bio-Oss, Geistlich, Switzerland) was placed in this area and also over the buccal in order to regenerate the dehiscence and in any other voids present where the roots were located (Fig. 12). Another collagen membrane was placed over the graft to protect it (Fig. 13).

Although our current approach is doing this technique in a single stage (using a healing abutment), in these kind of complex cases we decided to do it two stage due to the challenging situation. The primary closure was achieved with a coronal repositioned flap from the buccal (Fig. 14).

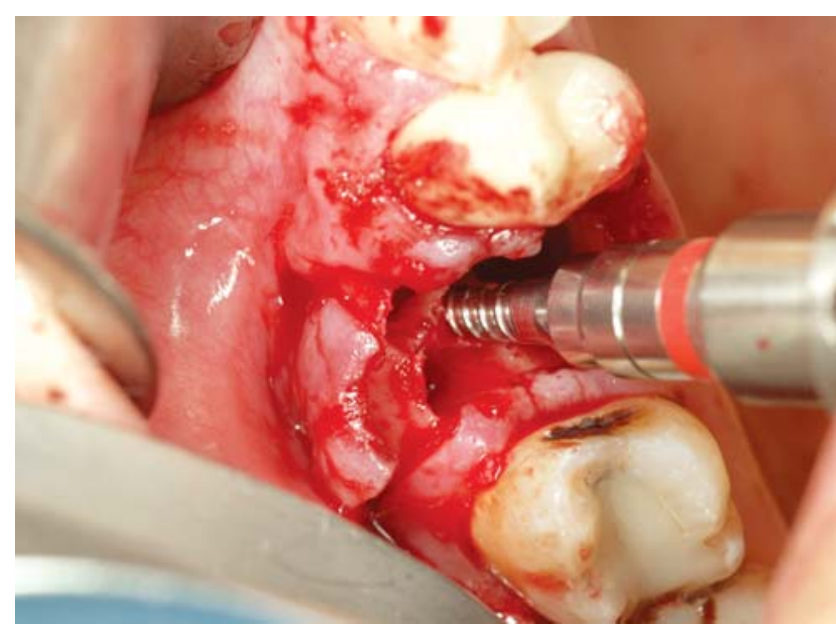

Fig. 8: Use of a pilot drill at the septal bone of the furcation, the expanders are used sequentially to develop the osteotomy

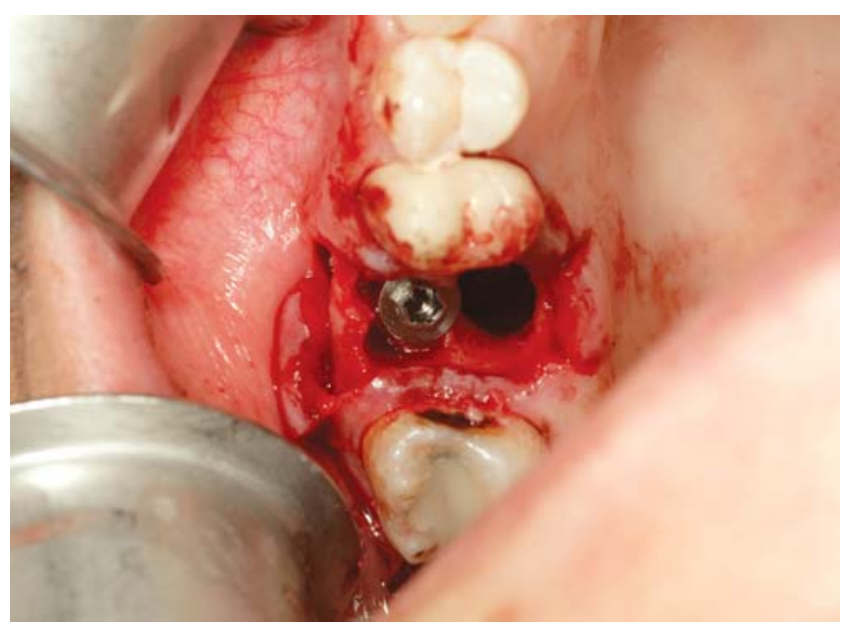

Fig. 9: Implant placed
After 4 months, second stage surgery was performed, and 15 days later the patient presents to initiate restoration of the implant (Fig. 15). The healing abutment is removed and an impression is taken following standard implant

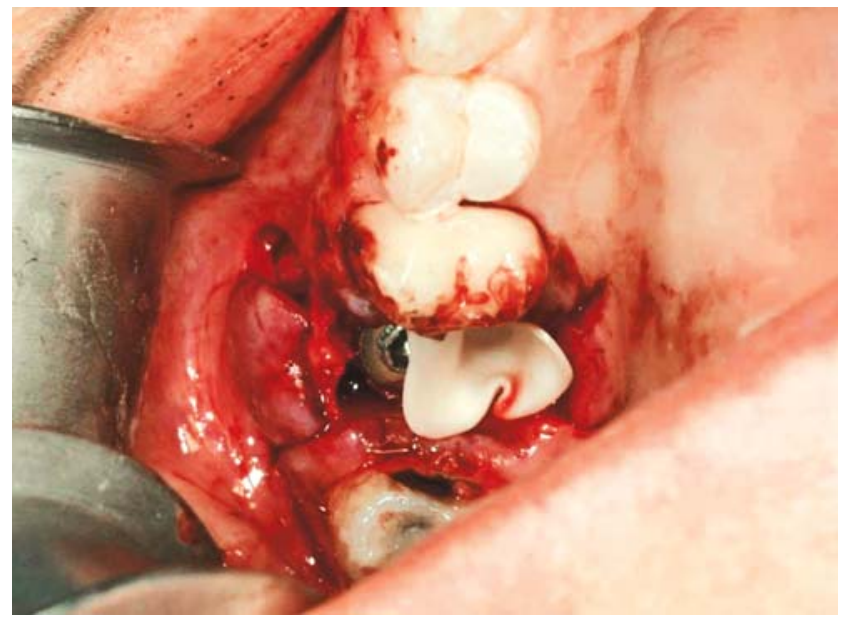

Fig. 10: Placement of a collagen membrane to close the maxillary sinus communication

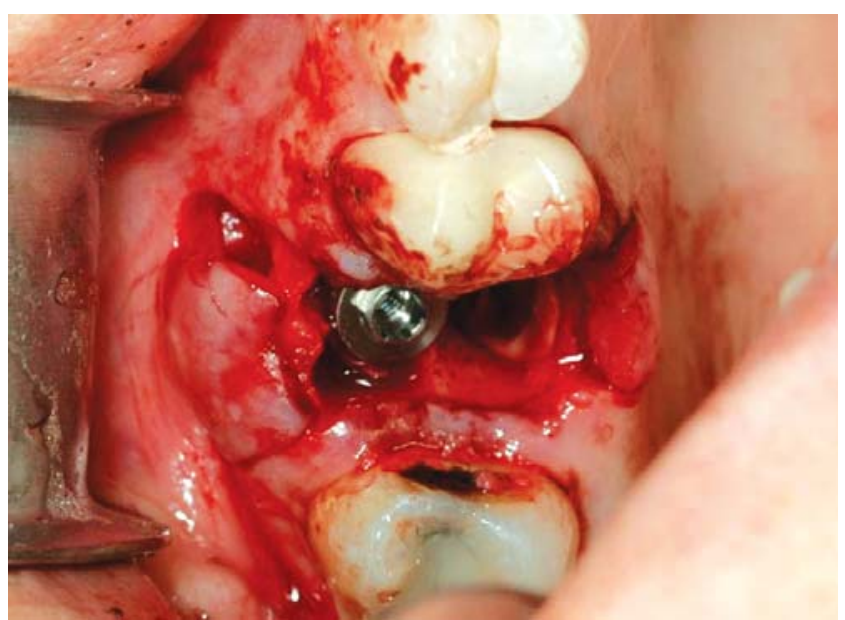

Fig. 11: Collagen membrane placed into the apical portion of the palatal root space where a sinus communication is present to act as a barrier to contain the graft to be placed

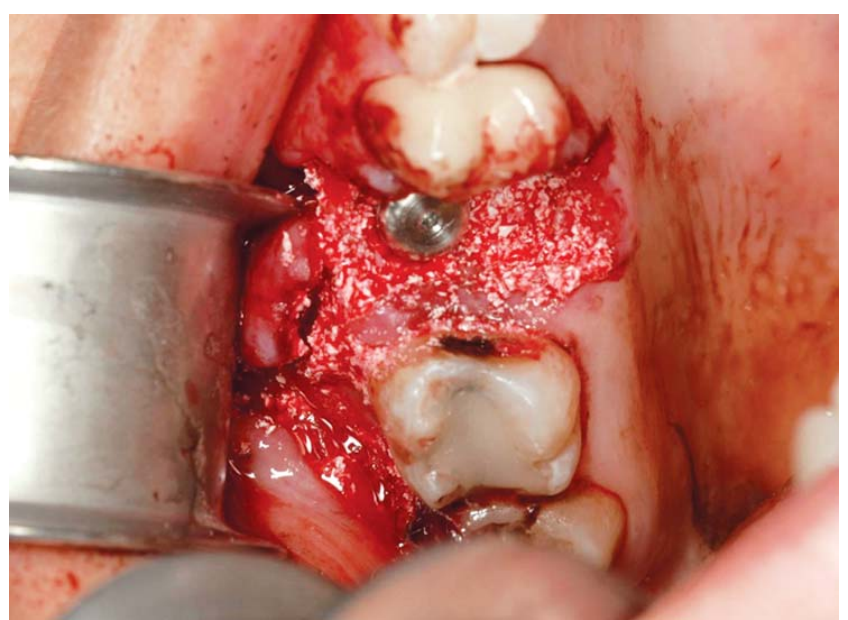

Fig. 12: Graft placed 
prosthetic principles. An abutment head is fabricated and a porcelain fused to metal crown is luted and occlusion checked and adjusted as appropriate (Figs 16 and 17). A

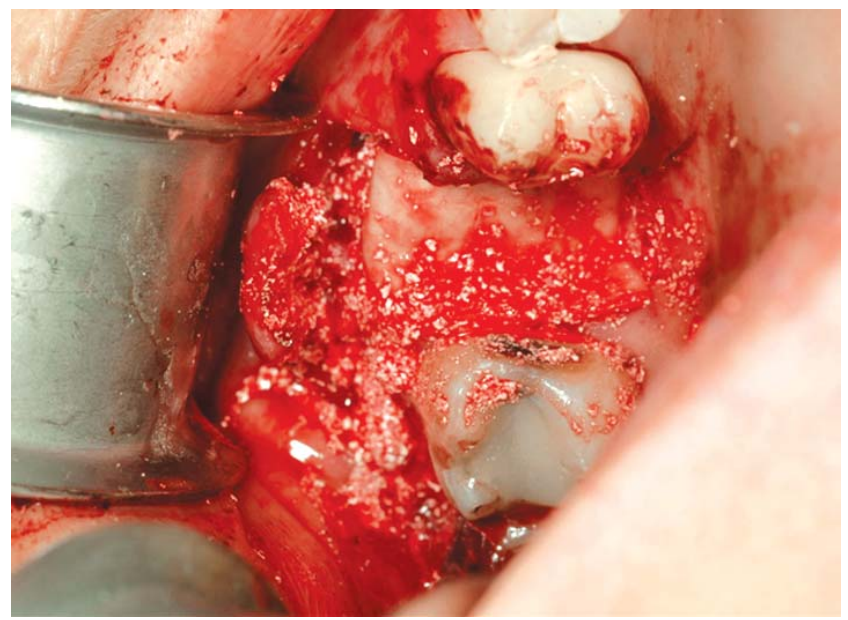

Fig. 13: Collagen membrane placed to protect the graft

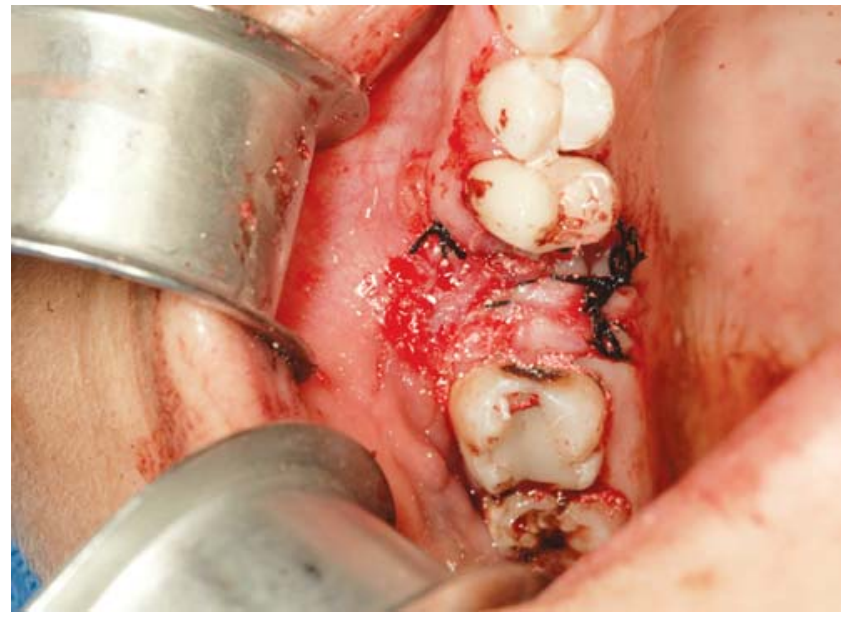

Fig. 14: The primary closure was achieved with a coronal repositioned flap from the buccal

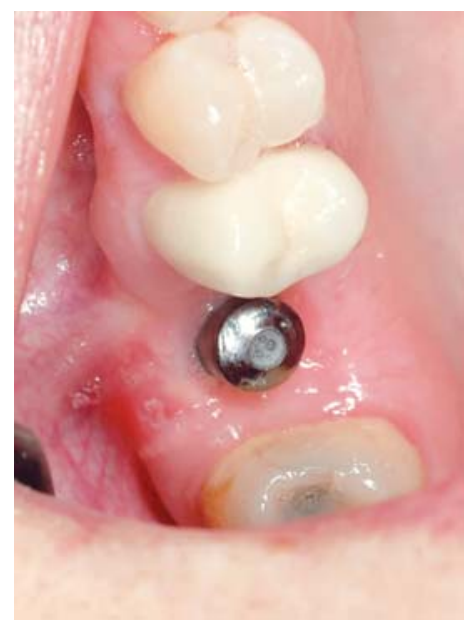

Fig. 15: Second-stage surgery, the patient presents for initiation of the prosthetics phase of treatment final radiograph is taken showing bone around the implant and fill of the root spaces present after extraction of the tooth (Fig. 18).

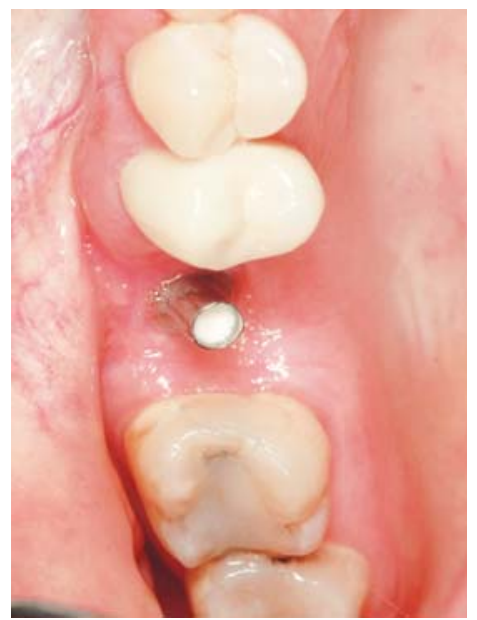

Fig. 16: Abutment in place

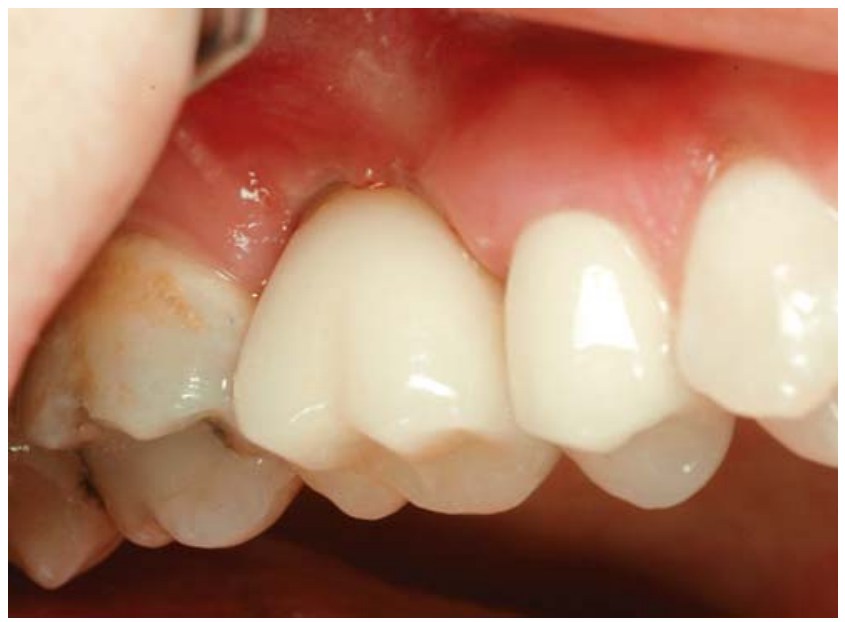

Fig. 17: Implant restoration on the maxillary first molar

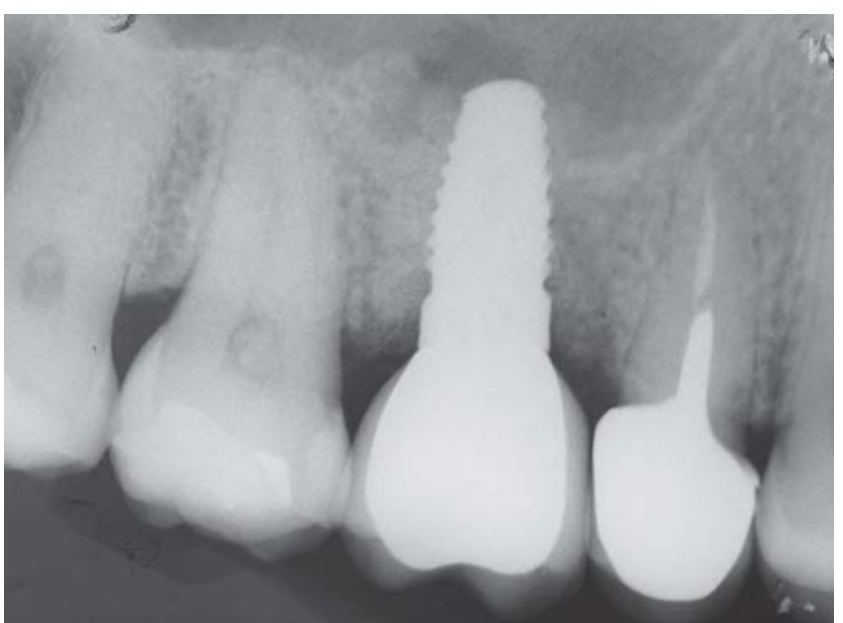

Fig. 18: Radiograph following restoration of the immediately placed implant at the maxillary first molar 


\section{Clinical Study}

Twenty-one patients, with an indication for maxillary molar extraction, were selected. Inclusion criteria included: Patients younger than 50 years old, tooth extraction due to caries, bone loss no greater than the coronal of the roots and with good general health. Implants were placed using threaded expanders following the technique described. Primary stability and osseointegration at second stage, 12 , 24 and 36 months were recorded by means of periotest device.

The periotest instrument measures the stability of the implant by percussing the fixture laterally and calculating the contact time. The result is then converted to a special scale called periotest value (PTV), which ranges from -8 to +50 . The lower the contact time the greater the stability and the lower the PTV. This translates to -8 representing the greatest stability and +50 the lowest. Usually, a PTV greater than +9 indicates a failing implant.

The results obtained were compared to the data of the authors' previous study in which they evaluated the technique using osteotomes and the delayed technique.

\section{RESULTS}

None of the implants placed failed during the evaluation period. PTVs were recorded at placement with values of $-2,476$ (0.68), and values of $-2,762(1.044)$ at second stage, $-2,857(0.655)$ at 12 months, $-3,429(0.507)$ at 24 months and $-3,476(0.512)$ at 36 months (Fig. 19).

Comparing these results with the previous data, we observed better results with the expander technique compared with the delayed technique, and no differences were observed with the osteotome technique. Combined data are shown in Table 1.

Statistical analysis was done using SPSS software. Oneway ANOVA was used for groups comparison at each measurement interval, and the Holm-Sidak method was used for all pairwise comparisons.

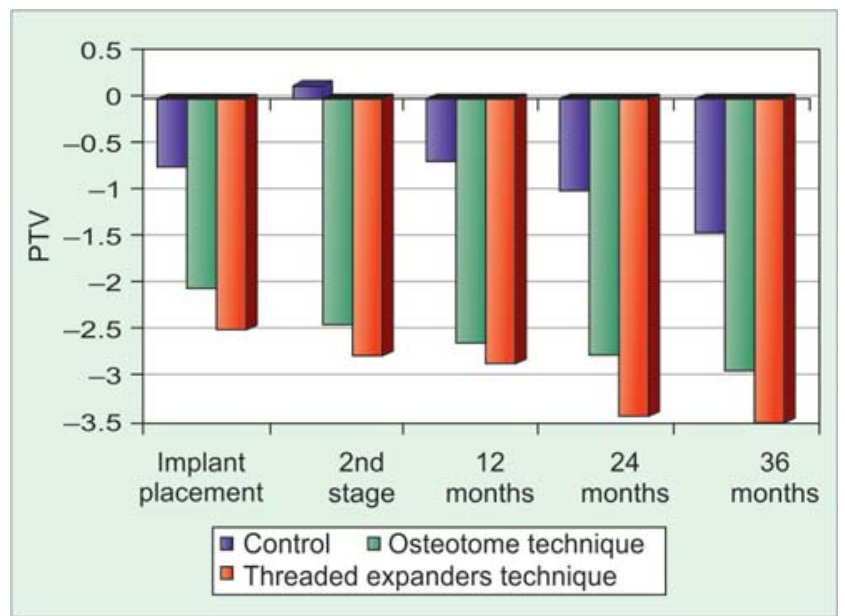

Fig. 19: Periotest results for the 21 patients observed

Statistical differences were found between the delayed technique and the two techniques for septa dilatation at initial placement, second stage, 12, 24 and 36 months ( $\mathrm{p}<0.001$ one-way ANOVA and $\mathrm{p}<0.05$ Holm-Sidak method). No differences were found between the two techniques for septa dilatation at each interval ( $p>0.05$ Holm-Sidak method).

\section{DISCUSSION}

The delayed technique has a few disadvantages, but longer treatment times are required and the possibility of bone loss during healing are the most often mentioned. ${ }^{10,11}$ We can add that the healed bone is usually softer than at the time of extraction and the height to the maxillary sinus is often less due to further pneumatization of the sinus. With this in mind, the site sometimes does not provide adequate conditions to achieve a good initial stability. Considering this, the delayed approach should be used only in case of active infection or in complex socket anatomy. However, as it was illustrated here, if the practitioner knows the technique, this can be performed also in complex cases.

Table 1: Mean PTVs and standard deviation of the different groups

\begin{tabular}{llrrrrr}
\hline & & $\begin{array}{c}\text { Implant } \\
\text { placement }\end{array}$ & 2nd stage & 12 months & 24 months & 36 months \\
\hline Contro/* $^{*}$ & Media & -0.727 & 0.136 & -0.682 & -1 & -1.455 \\
& SD & 1.352 & 0.889 & 0.894 & 0.873 & 1.011 \\
Osteotome technique & & $\mathrm{N}=22$ & $\mathrm{~N}=22$ & $\mathrm{~N}=22$ & $\mathrm{~N}=22$ & $\mathrm{~N}=22$ \\
& Media & -2.040 & -2.440 & -2.640 & -2.76 & -2.92 \\
& $\mathrm{SD}$ & 0.935 & 0.712 & 0.700 & 0.723 & 0.759 \\
Threaded expanders technique** & & $\mathrm{N}=25$ & $\mathrm{~N}=25$ & $\mathrm{~N}=25$ & $\mathrm{~N}=25$ & $\mathrm{~N}=25$ \\
& Media & -2.476 & -2.762 & -2.857 & -3.429 & -3.476 \\
& $\mathrm{SD}$ & 0.68 & 1.044 & 0.655 & 0.507 & 0.512 \\
& & $\mathrm{~N}=21$ & $\mathrm{~N}=21$ & $\mathrm{~N}=21$ & $\mathrm{~N}=21$ & $\mathrm{~N}=21$ \\
\hline
\end{tabular}

${ }^{*}$ Control and osteotome technique reproduced from Luchetti $\mathrm{C}$ and Kitrilakis A. ${ }^{8}$ New data from follow-up cases was added at 24 and 36 months, after this publication.

${ }^{* *}$ Threaded expanders technique at implant placement, 2nd stage and 12 months reproduced from Luchetti C, Kurtzman G, Kitrilakis A. ${ }^{9}$ 
The palatal socket off-axis technique is easy to perform and allows placement of longer fixtures. However, the implant placement leads to the need for angulation of the abutment for the restoration of the implant. Although there are no studies talking about bone loss in this situation, we can extrapolate of what is known, it is clear that we will have greater chances of failure. ${ }^{12,13}$ Another consideration in this approach is whether the implant is free standing or will function splinted with fixtures, which would better distribute occlusal loads and minimize the off-axis loading of the fixture placed into the palatal root of the extraction socket.

The palatal socket modification technique is a slightly more complicated, but still easy to perform. The approach is to enter in the palatal socket and change the trajectory of the drill making the fixtures axis more vertical. Placement of the implant is in the correct axis, but the future crown is situated more palatally. This may lead to a cantilever effect due to a portion of the crown laying buccal to the fixture. Additionally, hygiene problems may develop due to the over contour of the crown on the buccal.

The osteotome septa dilatation technique has shown good results, which have been better than the classic delayed approach and with shorter treatment time, as was shown in the authors previous study. ${ }^{8}$ When compared with other immediate techniques, although it is a more complicated surgically, it provides the buccal/palatal position and providing good axial loading of the future crown.

The threaded expander septa dilatation technique has also shown good results, being comparable to those placed with the osteotome technique regarding implant stability and positioning. Also, PTVs did not show statistical differences between the groups. However, the major advantage of the technique is that it is less traumatic to the patient, which may not be a minor issue. The risks of vertigo when using osteotomes has been reported in the literature. ${ }^{14,15}$ Although this risk is low and transient in nature, it is important to have this potential complication in mind with the use of osteotomes when there are no other options.

Threaded expanders are usually manually driven, using a thumb wrench or a raquet wrench. More recently, after our last article on the subject, we have incorporated the mechanical driven approach using a special adapter for the handpiece, which allows better control.

One of the potential problems when using the expanders manually is the risk to tilt the preparation mesially due to the direction of the rotational forces applied. The use of the mechanical approach has simplified this.

Also, the confidence gained with the technique allowed us to perform it in complex cases, as the one showed to illustrate the article, with good results. Although it is not the subject of the present article, immediate implant placement can be done even in cases with large apical infections, as is being demonstrated lately in the literature, including a paper from our group. ${ }^{16}$

\section{CONCLUSION}

The septa expansion technique has shown promising results. It is able to reduce the treatment time and provide better initial stability, allowing for better osseointegration in the long-term compared with the delayed technique. As was shown in the previous study, with the osteotome technique.

Immediate implant placement in maxillary molar sites is a predictable procedure. Within the immediate approaches, the septa dilatation technique seems to be the most adequate regarding buccal/palatal orientation and axial loading. Between the two techniques for septa dilatation, the threaded expander technique seems to be better, since it showed similar results to the osteotome technique, but was less traumatic for the patient. Further studies are needed to evaluate the other techniques.

\section{REFERENCES}

1. Lazzara RJ. Immediate implant placement into extraction sites: Surgical and restorative advantages. Int J Periodont Restorative Dent 1989;9:333-43.

2. Parel SM, Triplett RG. Immediate fixture placement: A treatment planning alternative. Int J Oral Maxillofac Implants 1990;5: 337-45.

3. Barizilay I, Grasser GN, et al. Immediate implantation of a pure titanium implant into an extraction socket: Report of a pilot procedure. Int J Oral Maxillofac Implants 1991;6:277-84.

4. Schwartz-Arad D, Gorssman Y, Chaushu G. The clinical effectiveness of implants placed immediately into fresh extraction sites of molar teeth. J Periodontal 2000;71:839-44.

5. Vergara JA, Caffesse RG. Immediate replacement of single upper posterior teeth: A report of cases. Clin Implant Dent Relat Res 2003;5(2):130-36.

6. Lim TJ, Csillag A, Irinakis T, et al. Intentional angulation of an implant to avoid a pneumatized maxillary sinus: A case report. J Can Assoc 2004 Mar;70(3):164-68.

7. Artzi Z, Parson A, Nemcovsky CE.Wide-diameter implant placement and internal sinus membrane elevation in the immediate postextraction phase: Clinical and radiographic observations in 12 consecutive molar sites. Int J Oral Maxillofac Implants 2003 Mar-Apr;18(2):242-49.

8. Luchetti C, Kitrilakis A. Immediate implant placement in the inter-septal bone of maxillary molars. J Indian Soc Oral Implantol 2006;3(1):1-5.

9. Luchetti C, Kurtzman G, Kitrilakis A. Immediate implant placement in maxillary molars using septa dilatation with threaded expanders. J Implant Advanced Clin Dent 2009; 1(4):19-28.

10. Schwartz-Arad D, Yaniv Y, Levin L, et al. A radiographic evaluation of cervical bone loss associated with immediate and delayed implants placed for fixed restorations in edentulous jaws. J Periodontol 2004 May;75(5):652-57. 
11. Penarrocha M, Uribe R, Balaguer J. Immediate implants after extraction. A review of the current situation. Med Oral 2004 May-Jul;9(3):234-42.

12. Canay S, Hersek N, Akpinar I, et al. Comparison of stress distribution around vertical and angled implants with finiteelement analysis. Quintessence Int 1996 Sep;27(9):591-98.

13. O’Mahony A, Bowles Q, Woolsey G, et al. Stress distribution in the single-unit osseointegrated dental implant: Finite element analyses of axial and off-axial loading. Implant Dent 2000; 9(3):207-18.

14. Flanagan D. Labyrinthine concussion and positional vertigo after osteotome site preparation. Implant Dent 2004 Jun;13(2): 129-32.

15. Peñarrocha-Diago M, Rambla-Ferrer J, Perez V, Pérez-Garrigues $\mathrm{H}$. Benign paroxysmal vertigo secondary to placement of maxillary implants using the alveolar expansion technique with osteotomes: A study of 4 cases. Int J Oral Maxillofac Implants 2008 Jan-Feb;23(1):129-32.

16. Luchetti C, Kitrilakis A. immediate dental implants in infected sockets. Microbiological aspects, marginal bone level and implant stability. Clin Oral Implants Res 2010;21(10):1038, a118.

\section{ABOUT THE AUTHORS}

\section{Cesar G Luchetti (Corresponding Author)}

Associate Professor, Department of Implant Dentistry, National University of La Plata, Buenos Aires, Argentina, e-mail: luchetti. implantlist@gmail.com

\section{Gregori M Kurtzman}

Private Practice, Silver Spring, Maryland, USA

\section{Alicia E Kitrilakis}

Professor and Head, Department of Implant Dentistry and Prosthodontics, National University of La Plata, Buenos Aires Argentina

\section{Daniel I Ostrowicz}

Private Practice, Barcelona, Spain 